\title{
Communication interne : une expérience de détaylorisation croisée
}

Jacqueline Cons-Soubeiran

\section{(2) OpenEdition}

1 Journals

Édition électronique

URL : http://journals.openedition.org/communicationorganisation/1845

DOI : 10.4000/communicationorganisation. 1845

ISSN : $1775-3546$

Éditeur

Presses universitaires de Bordeaux

Édition imprimée

Date de publication : 1 mai 1996

ISSN : 1168-5549

Référence électronique

Jacqueline Cons-Soubeiran, "Communication interne : une expérience de détaylorisation croisée », Communication et organisation [En ligne], 9 | 1996, mis en ligne le 26 mars 2012, consulté le 01 mai 2019. URL : http://journals.openedition.org/communicationorganisation/1845 ; DOI : 10.4000/ communicationorganisation. 1845

Ce document a été généré automatiquement le 1 mai 2019.

(c) Presses universitaires de Bordeaux 


\title{
Communication interne: une expérience de détaylorisation croisée
}

\author{
Jacqueline Cons-Soubeiran
}

1 La communication interne n'en finit pas d'être, au fil des constats, qualifiée de volontariste et unilatérale, d'oublier les attentes des salariés malgré toute la panoplie d'outils dont elle dispose.

2 Une expérience professionnelle récente, la direction de l'organisme de formation d'un groupe important en pleine restructuration, m'a permis de participer à une démarche impulsée par les Directeurs de Ressources Humaines du groupe et visant à dépasser ce constat récurrent. Cette expérience est la tentative de mise en place d'une communication interne participative ayant choisi la formation comme terrain d'expérimentation. Il s'agissait de donner à la communication interne la possibilité d'élargir ses objectifs et ses modalités d'action, et de les consolider, en faisant participer les collaborateurs de l'entreprise, à tous les niveaux, à la conception et à la mise en œuvre des actions de communication et de formation.

3 La relation de l'expérience fera l'objet des deux premières parties de l'article :

4 - Le contexte à l'origine de l'élaboration d'un nouveau processus de communication lié à l'ampleur des restructurations, la volonté d'alliance de deux acteurs-clés: le Comité Ressources Humaines et l'organisme de formation, seront abordés dans un premier temps. La complexité du groupe, les positions respectives du CRH et de l'organisme de formation, rendent indispensable une telle clarification.

5 - La mise en place, par les deux partenaires, d'un processus de communication participatif expérimenté à travers le renouveau des objectifs et des actions de formation du groupe sera ensuite retracée.

6 La troisième partie permettra de poser sur cette expérience professionnelle, un regard critique éclairé par la lecture de chercheurs en sciences de l'information et de la communication, et de sociologues des organisations et de la formation. L'analyse sera centrée sur l'évolution des pratiques de communication et de formation en entreprise. 


\section{Le contexte à l'origine de l'élaboration d'un nouveau processus de communication}

7 Depuis 1990, le groupe Palme ${ }^{1}$, important groupe d'assurances, vit, pour faire face à la concurrence, une période de restructurations difficiles à maîtriser. Malgré l'existence d'une direction de la communication très équipée mais diffusant des messages à sens unique : du haut de la pyramide vers la base, ces restructurations sont vécues comme un diktat. Dans ce contexte, deux acteurs ${ }^{2}$ clés du changement dans l'entreprise se positionnent pour essayer de mettre en place un processus de communication participatif.

\section{Des restructurations difficiles}

8 Le groupe Palme est composé jusqu'en 1990 de 70 entreprises départementales ou pluridépartementales réparties sur l'ensemble de la France. Des sociétaires élus constituent les conseils d'administration de chaque entreprise. En 1990, pour préserver ses marchés face aux autres groupes d'assurances, mieux compenser les risques, pratiquer une gestion et une distribution plus performante, les dirigeants du groupe Palme décident de rassembler les entreprises départementales en 18 entreprises régionales.

9 Cette exigence initiale sera progressivement assouplie sous la pression des circonstances. En effet, les regroupements d'entreprises, préparés de façon hâtive et à partir de critères presque exclusivement quantitatifs (montant des encaissements) posent de nombreux problèmes. Les problèmes de répartition de pouvoir dominent :

10 - au niveau régional d'une part: passer de 70 conseils d'administration et directeurs généraux à 18 ne peut se faire sans heurts ni luttes de personnes,

11 - entre l'organisme central et les entreprises régionales d'autre part. Celles-ci ont tendance à devenir des «Baronnies» d'autant plus réelles, que seuls des pouvoirs régionaux puissants arrivent à imposer sur le terrain les regroupements décidés et parfois impopulaires.

12 Les tensions sont fortes. Elles alimentent une rumeur et une rancœur lancinantes, d'autant plus que les résultats du groupe sont menacés par une concurrence de plus en plus vive et que les restructurations coûtent cher. Pour tenter de fédérer cet ensemble agité, des comités fonctionnels sont mis en place: comité technique, commercial, financier, comité ressources humaines. Chacun de ces comités est composé des hommes responsables de la fonction considérée, dans les entreprises régionales. Des directeurs généraux d'entreprises régionales président chaque comité.

13 Très vite, chaque comité devient un lieu d'affrontement. S'il n'y a pas éclatement réel du groupe, il y a risque de blocage dû à la difficulté à définir des stratégies communes. Et cela pour des raisons diverses, parfois contradictoires: désaccord avec le projet de regroupement, mêlé à la peur d'avoir à gérer des entreprises beaucoup plus importantes, rivalités de personnes, amertumes liées à la configuration des regroupements.

14 Une communication interne mécanisée au centre de laquelle le salarié paraît complètement oublié3. 
15 De nombreux outils de communication existent dans le groupe : journaux d'entreprise, revues de presse, conventions annuelles (réservées aux cadres), assemblées générales animées à l'aide de moyens audiovisuels sophistiqués. Il s'agit presque toujours de transmettre les messages de la direction «en langue de coton »"c'est-à-dire de transmettre une information qui ne dérange pas et qui soit systématiquement positive.

Ce qui s'est passé à Air France montre combien l'utilisation d'outils de communication sophistiqués cohabite fort bien avec l'absence d'écoute et de dialogue.

17 À ce moment de l'histoire du groupe, le système de communication qu'est l'entreprise, selon l'expression de Philippe SCHEWIG ${ }^{5}$ qui sera développée plus loin, fonctionne de façon instable et souvent négative. À tous les niveaux hiérarchiques, on se plaint. «Les regroupements nous coûtent cher, nous opposent, absorbent notre énergie, nous font reculer sur les marchés ». À l'échelon central, on déplore de ne plus rien maitriser. En région, on s'insurge «nos particularités régionales ne sont pas reconnues». En fait, ce qui s'exprime, c'est un sentiment d'impuissance généralisée, lourde de conséquence pour l'avenir : déperdition du sentiment d'appartenance/groupe, risque de pertes de marché, insécurité.

\section{La prise de position du Comité Ressources Humaines soucieux d'initier une communication interne participative.}

18 Le comité RH, composé de personnes qui, de par leur fonction, sont sans doute plus conscientes des enjeux de l'avenir, souhaite inverser la tendance négative et destructrice de ce système de communication instable. Il décide d'essayer de créer les conditions d'un courant positif et structurant en agissant dans les domaines vitaux qui sont les siens (évolution de l'emploi, des compétences, formation). L'objectif est, notamment, de montrer qu'il est possible dans le cadre des nouvelles structures, d'agir ensemble, après avoir «mis en commun». Il s'agit, par l'exemplarité de cette action, de «rendre communicante la structure organisationnelle ${ }^{6} »$.

19 Les DRH vont essayer d'assurer une fonction de communication interne ascendante et descendante où il y ait réellement échange, où les messages venant de la base soient entendus.

20 Le CRH va choisir un terrain d'action où le changement de processus, "la détaylorisation ${ }^{7}$, c'est-à-dire la démarche visant à tenter de lever les barrières entre conception et exécution, renforce, crédibilise son propre processus de communication et d'action, que l'on peut aussi qualifier de " détaylorisation " puisque les premières étapes d'une démarche participative sont en train de s'y élaborer : les représentants de chaque entreprise régionale décident en commun à partir des informations et avis recueillis dans leur région, auprès de leurs directions, des syndicats et des salariés dont ils assurent la gestion et l'évolution. Ils appliquent ensuite dans leur entreprise ce qui est décidé en commun.

21 Le terrain d'action choisi sera la formation et le partenaire avec lequel il y aura alliance, l'organisme de formation du groupe : l'OFOR $^{8}{ }^{\prime}$. 


\section{L'alliance de deux acteurs-clés.} permettant aux dites structures de communiquer.

\section{La mise en place d'un processus de communication et d'action participatif qui débouche rapidement sur l'action-formation.}

\section{L'élaboration d'un nouveau processus.}

\section{Nouveaux acteurs et nouvelles modalités de communication}

27 Le comité RH accueille à sa demande, comme membre à part entière, le nouveau responsable de l'organisme de formation du groupe, dès octobre 1993. Cette " participation » s'est opérée de façon très démocratique, puisque tous les membres du groupe y étaient expressément favorables. 
28 Le CRH va pouvoir vérifier l'efficacité de ses modalités participatives de fonctionnement en les appliquant à la réflexion et à l'action Formation. On peut les schématiser ainsi : groupe de travail fi présentation des résultats en grands groupes fi discussion fi décision. Il n'y a pas de sous-groupe Formation figé une fois pour toutes : tous les DRU participent successivement aux différentes étapes d'avancement. Il y a donc réellement «mise en commun ", communication entre tous les membres du groupe. Ceux-ci sont par ailleurs les relais de communication ascendante et descendante avec leurs entreprises. Ils présentent en effet régulièrement les étapes d'avancement des travaux en cours, au comité de direction de leur entreprise, aux services concernés et aux responsables de formation. Les réactions et suggestions remontent par la même voie au CRH.

\section{Mise en œuvre du processus pour redéfinir la formation et ses modalités de conception et réalisation.}

29 La finalité de la formation du groupe sera d'aider les entreprises et donc leurs salariés à s'adapter à l'évolution des emplois imposée par un contexte économique plus concurrentiel.

30 Pour atteindre cet objectif, plusieurs points seront successivement abordés : la définition des actions prioritaires, les modalités participatives d'élaboration des actions de formation et les modalités participatives de mise en œuvre de ces actions.

31 - La définition des actions et des cibles prioritaires : il faut "professionnaliser" les salariés, l'ensemble des salariés et notamment les salariés dits "d'exécution » gestionnaires et commerciaux, les recentrer sur leur métier: l'assurance. Il faut également assurer l'intégration de cette formation en professionnalisant d'abord les cadres de proximité. (Il n'y a pas vraiment de niveau maîtrise).

32 - Les modalités participatives d'élaboration des actions de formations: au delà des groupes de travail du comité RH, des comités de pilotage seront créés pour les différents types d'action, faisant appel à la fois aux hiérarchies des entreprises concernées et aux salariés destinataires de l'action. Des enquêtes réalisées dans les entreprises permettront d'affiner les objectifs généraux et pédagogiques de chaque formation, et de mettre en commun en les intégrant dans les formations, les savoir-faire « exemplaires » des salariés du groupe.

33 - Les modalités participatives de mise en œuvre des actions de formation et de leur suivi : elles passent par l'implication directe des hiérarchies et des individus.

34 - Implication directe des hiérarchies : sélection des stagiaires liée au projet professionnel, intervention dans les formations, suivi.

35 - Implication des individus : choix de la formation intégrée au projet professionnel, après entretien avec la hiérarchie, le responsable de formation et le DRH de l'entreprise. L'évaluation des formations et le suivi seront réalisés au niveau de l'entreprise par les mêmes partenaires que ceux précédemment cités. Ces informations remonteront ensuite au comité RH grâce au rôle d'interface de l'OFOR.

36 Pour soutenir l'ensemble du processus, il est nécessaire d'en professionnaliser un relais essentiel : les responsables de formation : des journées de formation/métier sont créées. Elles serviront en outre de vecteur privilégié de communication ascendante et descendante. 
37 La conception et la mise en œuvre d'actions de formation détaylorisées, semblent crédibiliser le nouveau processus.

Une première réalisation : un cycle long de formation pour les cadres de proximité. promotion d'« objectif cadre » démarre avec 20 stagiaires. Dès juillet 1995, d'après les responsables de l'entreprise et les stagiaires eux-mêmes, ceux-ci auraient progressé dans leur métier à travers une amélioration de leurs compétences techniques et relationnelles. L'évaluation est encore peu formalisée. Elle est réalisée à travers une série d'entretiens dans l'entreprise à l'issue desquels sont remplis des questionnaires d'évaluation très ouverts. 
processus de communication, puisque la démarche participative va jusqu'au bout de sa logique.

51 Le perfectionnement du processus de communication permet d'envisager la réalisation d'actions plus lourdes intégrant dans leur conception les usagers/destinataires de la formation.

\section{L'action « gestionnaires »}

52 Le projet est de former 3000 gestionnaires d'assurance à l'évolution de leur métier : évolution technique et relationnelle.

53 La démarche participative est plus complète dès la phase de conception :

54 - création d'un comité de pilotage en juin 1995 (DRH + OFOR + Techniciens), création d'un groupe de conception avec des gestionnaires d'assurance travaillant dans les entreprises régionales ;

55 - étude d'un référentiel de compétences réalisée dans les entreprises régionales en août 1995. Il s'agit de mieux définir les compétences actuelles et les compétences futures requises par l'évolution du métier. Cette étude sera réalisée au moyen d'une série d'entretiens dans des entreprises régionales sélectionnées à partir du caractère représentatif des gestionnaires d'assurance y travaillant. Elle fait ressortir la nécessité de donner aux gestionnaires d'assurance une vision globale et unitaire de leur métier dont les deux composantes sont la production de contrats et la gestion de sinistres. Cette vision globale requiert un niveau plus élevé de connaissances techniques et une meilleure intégration du métier à la démarche commerciale globale de l'entreprise.

56 Les modalités participatives envisagées pour la mise en œuvre de la formation sont également plus audacieuses :

57 - compte tenu de la masse importante de gestionnaires à former, des particularités des entreprises régionales, de la nécessité d'intégrer les cadres de l'entreprise à la formation elle-même pour mieux en assurer le suivi au poste de travail, la formation sera très décentralisée.

58 - Le groupe de conception qui démarre en octobre 1995 en liaison avec le comité de pilotage, décide d'élaborer un ensemble pédagogique multimédia très constuit et détaillé, permettant de réaliser les formations dans chaque entreprise; ces formations seront animées par des cadres de l'entreprise en co-animation : un animateur professionnel, un formateur assurant l'animation des parties techniques, (cadre technique, si possible le supérieur hiérarchique). La formation "gestionnaire » est testée et finalisée en mai 96 dans une entreprise régionale.

59 La communication interne mise en place soutient le projet et en assure le préfinancement.

60 - Une information sur le projet et son évolution est régulièrement transmise par les DRH et l'OFOR aux directeurs généraux, directeurs techniques et responsables de formation: lettres d'information régulières, informations orales données dans chaque entreprise par les DRH, au cours de réunions de direction ou de réunions de services. Les entreprises régionales et leurs cadres sont par ailleurs directement impliqués dans le processus de mise en place de la formation puisqu'ils se constituent dans chaque entreprise en comité de pilotage. Leur mission est d'informer les salariés de l'entreprise, d'organiser les étapes 
préalables à la formation et la formation elle-même avec les cadres responsables des unités de gestionnaires d'assurance.

61 - Un référendum «financier »: l'OFOR ne pouvait financer un projet aussi lourd. En octobre 1995 l'organisme présente, par écrit, à chaque directeur régional, le projet avec ses objectifs et les moyens envisagés pour sa mise en œuvre: moyens pédagogiques, organisationnels et financiers. Il est proposé à chaque directeur régional de concrétiser son accord sur le projet en s'engageant à le financer. Un contrat définit les modalités et les échéances de cet engagement. Le résultat est surprenant : chaque entreprise régionale préfinance le projet et s'engage donc par contrat à acheter et mettre en place la formation " gestionnaire » dans les conditions définies. Cette " participation financière " semble bien être le fruit de l'effort de participation et de communication de tous les acteurs.

\section{Le projet de formation commerciale}

62 L'action gestionnaire d'assurance a permis, semble-t-il, d'atteindre l'objectif d'exemplarité de la démarche utilisée : en juin 1996, le comité fonctionnel commercial composé de directeurs commerciaux des entreprises du groupe demande au CRH et à l'OFOR de concevoir et réaliser une action de formation pour tous les commerciaux du groupe, en commençant par la base : les salariés travaillant sur le terrain. Le principe d'une démarche participative est souhaité, de la conception au suivi, intégrant toute la filière commerciale: commercial terrain fi responsables de secteurs fi directeurs commerciaux.

Cette demande est importante: les directeurs commerciaux du groupe tentent, depuis deux ans, avec difficulté, de définir ce qu'ils appellent « un langage commun » pour leur réseau de vente, afin de renforcer l'impact sur des marchés qui se globalisent. Leur demande est la reconnaissance de l'efficacité d'une démarche dont les caractéristiques participatives leur permettront, enfin, de faire le point, à partir d'enquêtes sur le terrain, sur l'évolution nécessaire des compétences de leur réseau, tout en motivant ce dernier et en le rendant acteur de son propre changement.

C'est aussi l'affirmation d'une volonté de ne plus considérer les différentes fonctions de l'entreprise comme autant de territoires distincts et en partie étanches et d'instaurer avec l'aide des DRH une véritable communication entre les fonctions et à l'intérieur de chacune d'entre elles, afin d'assurer un développement cohérent de leur entreprise. La conviction d'être porteurs d'une communication interne renouvelée est telle, qu'il est prévu, à l'occasion de ce projet de formation commerciale, la création d'un journal par le $\mathrm{CRH}$, le comité commercial et l'OFOR ; l'objectif est d'informer les entreprises régionales et leurs salariés de l'évolution des formations mises en place, reliées à l'évolution des marchés de l'assurance, aux nouveaux produits,...

\section{Regard critique}

Portons sur cette expérience un regard aiguisé par la lecture de chercheurs en sciences de l'information et de la communication et de sociologues de l'organisation et du travail.

L'expérience pourrait être analysée à travers différentes approches: celle de l'analyse stratégique et des jeux de pouvoir de nouveaux acteurs «profitant» d'une zone 
d'incertitude, la problématique de l'innovation, de sa protection et de sa formalisation éventuelle...

Toutefois, l'objectif opérationnel étant la mise en place d'une communication interne participative à partir du choix de la formation comme terrain d'expérimentation, il parait plus opportun de centrer l'analyse sur l'évolution des pratiques de communication et de formation en entreprise. Nous pourrons ainsi essayer de situer l'expérience par rapport aux évolutions repérées et analysées dans ces deux domaines d'activités.

Après avoir, sous cet angle, étudié le contexte dans lequel l'action des DRH s'est inscrite et plus particulièrement l'inefficacité de la communication interne officielle, nous aborderons les caractéristiques de cette action et notamment le choix du terrain d'expérimentation.

Nous nous attacherons enfin, de façon plus détaillée, à cerner l'évolution des formations conçues et réalisées qui pourraient s'inscrire dans les formations dites détaylorisées, sur lesquelles ont travaillé plusieurs chercheurs en sociologie des organisations et du travail.

\section{«L'introuvable « communication interne}

Le contexte dans lequel s'inscrit l'expérience relatée est bien celui des difficultés de la communication officielle, particulièrement évidente en cas de crise.

71 Il semble en effet que l'on se trouve devant l'une des situations souvent décrites par les sociologues des organisations, qui ont amené Michel Crozier à porter un jugement sévère bien connu : «Si la communication interne aboutit à un résultat nul, c'est... parce qu'elle est presque toujours conçue comme un moyen de faire passer des messages du sommet de la pyramide vers le bas'... ».

72 L'incompréhension des salariés du groupe face à la nécessité affirmée des regroupements qui suscite à tous les niveaux les réactions négatives décrites en lère partie,prouve l'absence de dialogue, d'écoute et tout simplement d'explication un peu approfondie prenant en compte les interrogations des salariés.

73 Il paraît vital, devant un tel changement, si mal vécu, d'essayer de maîtriser au moins pour partie, le système de communication que devient l'entreprise dans un tel contexte, comme le décrit Ph. Schwebig. « Un environnement incertain et en perpétuelle mutation, les ruptures techniques et technologiques, la globalisation des marchés, les aspirations profondes et irrésistibles du corps social vers le participant ont transformé l'entreprise en un véritable système de communication ${ }^{10}$."

74 C'est le constat fait par les DRH des différentes entreprises du groupe Palme et la raison d'être de leur tentative d'assurer une fonction de communication interne qui sorte de l'unilatéralité existante et tente d'être comme l'écrit C. Le Moenne «comprise comme capacité d'écoute et de dialogue et capacité d'intermédiation entre la hiérarchie supérieure et la base ${ }^{11} »$.

75 Le comité RH semble présenter les composantes principales de la communication interne, rappelées par F. Charvin ${ }^{12}$ :

76 - Il est au niveau du groupe, un système interne d'écoute de par les hommes et femmes qui le composent. Les DRH peuvent recueillir les avis et les positions de leurs directions, des syndicats et des salariés dont ils assurent la gestion et l'évolution. 
77 - Il est l'expression interne de l'identité du groupe dans sa complexité puisque chaque région est représentée avec sa problématique région/groupe, appartenance, indépendance.

78 Par ailleurs le fait, pour les DRH, d'essayer de mettre en place de telles démarches de communication va dans le sens d'une évolution qui semble se dessiner dans les entreprises françaises; selon une enquête récente, $51 \%$ des services de communication interne sont rattachés aux DRH. Le constat est présenté ainsi : "c'est la nouveauté de l'enquête, les services de communication sont de plus en plus aujourd'hui rattachés au service des ressources humaines... on peut donc augurer à plus ou moyen terme une communication plus sociale ${ }^{13} »$.

\section{Le choix d'un terrain d'action significatif de la volonté de changement et de participation permettant de « construire " un consensus, s'appuie sur de nouvelles alliances.}

79 La formation est reconnue par de nombreux auteurs comme un des moyens privilégiés du changement dans l'entreprise et de l'expression d'une volonté de participation.

80 À côté des techniques de communication, B. Miège met en valeur la formation comme une des techniques qui participent «tout particulièrement » à la mise en place de nouvelles modalités de travail ${ }^{14}$. Le sociologue R. Sainseaulieu attribue d'ailleurs à la formation une place privilégiée puisqu'il la décrit comme "porteuse, à travers ses actions, de processus transformateurs et de changements organisationnels suffisants pour que l'on puisse la ranger au nombre des voies privilégiées du développement social des entreprises contemporaines ${ }^{15} »$.

81 Choisir comme terrain d'expérimentation d'une communication interne participative la formation, paraît donc un choix judicieux. D'autant plus qu'elle est, par elle-même, un lieu de communication et de participation. R. Sainseaulieu, toujours attaché à mettre en valeur la formation, insiste sur ces deux points : après avoir précisé que « les stages de formation s'avèrent être, dans l'ordre des communications, d'excellents lieux de passage d'information inter-services et même inter-entreprises ${ }^{16}$ ", il ajoute que «le développement de la formation des adultes peut être considéré comme porteur d'un plus fort degré de participation dans les structures ordinaires d'organisation du travail ${ }^{17}$ ».

Dans l'expérience relatée, l'intervention des cadres experts du groupe choisis en raison de leurs compétences professionnelles et pédagogiques permet l'information et la discussion sur l'évolution de l'entreprise, de ses marchés, et de ce fait des nouvelles exigences en matière d'organisation du travail: liens nécessaires entre les services techniques et les services commerciaux, polyvalence mise progressivement en place pour la gestion des contrats d'assurance, rôle relationnel accru des responsables d'équipe.

83 Par ailleurs la formation est une forme d'action plus facile et plus rapide à concevoir et à mettre en œuvre qu'un gros chantier comme celui d'une convention collective. Or les DRH avaient besoin d'une action qui leur permette de crédibiliser leur fonctionnement consensuel qui ne se décrète pas, mais, comme l'affirme $\mathrm{M}$. Crozier, est le résultat d'une action et «non pas un préalable à l'action ${ }^{18}$ ».

84 Le contexte du groupe Palme, semblait imposer cette nécessité d'agir rapidement et de montrer qu'une démarche de communication interne participative n'était pas un vœu pieu mais permettait d'obtenir des résultats. 

travaillant sur l'évolution de la formation et notamment par $\mathrm{N}$. Bousquet et $\mathrm{C}$. Grandgérard. La caractéristique principale du taylorisme est la division du travail basée sur une séparation nette entre les fonctions de conception et les tâches d'exécution, séparation qui ne permet pas de tirer parti des nombreuses informations détenues par les salariés d'exécution. Détayloriser, c'est dépasser cette division du travail organisée au sommet, lever les barrières entre conception et exécution, c'est donc mettre en place, pour la communication comme pour la formation, des modalités participatives intégrant les destinataires finaux. I'OFOR, étaient conçues de façon centralisée à l'aide de consultants extérieurs, mais sans la participation active de membres des entreprises du groupe. Ces formations étaient vendues par catalogue, sans possibilité pour le destinataire de les modifier. Nous sommes dans le cas, comme l'écrit $\mathrm{M}$. Feutrie, «d'une relation à partir d'un catalogue, d'une logique de construction dont la conception ou l'initiative appartiennent à une instance extérieure $^{21} »$.

N. Bousquet et C. Grandgérard déterminent quatre critères " matérialisant » la recherche de détaylorisation dans le domaine de la formation: la notion de masse critique, de continuum, la durée, la formation, projet négociéé2 .

91 Les modalités participatives de conception, d'élaboration et de suivi des actions de formation mises en place en 1994 par les DRH et I'OFOR, semblent avoir permis la réalisation d'actions de formation répondant, pour l'essentiel, aux conditions déterminées par N. Bousquet et C. Grandgérard.

\section{La notion de masse critique}

Pour permettre aux salariés d'une entreprise d'être acteurs du changement, il est nécessaire que le plus grand nombre d'entre eux bénéficient des formations indispensables à l'évolution de l'entreprise. 
Dans l'expérience relatée, 3000 gestionnaires d'assurance et 1500 commerciaux sont les destinataires de deux des formations réalisées ou en cours de réalisation.

\section{La notion de continuum}

Il ne suffit pas d'investir dans des formations de masse pour créer les conditions d'un changement qui bénéficie de l'évolution du potentiel de toute l'entreprise. Il faut également favoriser la création d'une chaîne continue de formation allant du salarié de base aux différents niveaux d'encadrement (intégration verticale). Cette notion de continuum s'applique également à la formation de fonctionnels de même niveau hiérarchique (intégration horizontale).

Dans l'expérience décrite, le continuum "vertical» est en cours de réalisation: la formation des cadres de proximité, gestionnaires ou commerciaux a été envisagée comme objectif prioritaire, en même temps que les formations du personnel dit "d'exécution ", afin de favoriser l'intégration des formations de ces deux niveaux hiérarchiques. « Objectif cadre » lancé volontairement un an avant " gestionnaire» doit permettre aux cadres de proximité de participer par la suite à la formation de leurs équipes de gestionnaires.

Par ailleurs, l'intervention, dans les formations citées, des différents niveaux de cadres hiérarchiques, appelés «Experts» techniques ou commerciaux semble également correspondre à la notion de continuum vertical.

7 Le continuum horizontal n'est pas encore réalisé, mais la concertation permanente entre les DRH, les directeurs techniques et les directions membres du comité de pilotage responsable de l'implantation dans chaque entreprise de la formation "gestionnaire ", peut être considérée comme une étape préalable au continuum horizontal.

\section{La durée}

Cette condition, telle qu'elle est présentée par N. Bousquet et C. Grandgérard, met en valeur la nécessité, pour les formations de ne pas être coupées de l'évolution du travail lui-même, d'être «des étapes dans un processus global et systémique dans lequel des organisations constamment évolutives appellent toujours plus de connaissances, d'autonomie et d'adaptabilitéé ${ }^{2} »$.

"Objectif cadre", formation des cadres de proximité, s'étale sur vingt mois, en alternance avec l'activité professionnelle stricto sensu. À l'issue de la formation, des cercles de progrès annuels sont envisagés.

Quant à la formation "gestionnaire ", plus courte, 12 jours répartis sur 5 à 6 mois, elle doit faire l'objet d'un suivi systématique au poste de travail, par les cadres hiérarchiques (qui participent à la formation) et par le comité de pilotage de l'entreprise. Par ailleurs, le contenu de la formation est l'objet d'une réactualisation permanente, prévue par le contrat de départ.

1 Inscrites donc dans la durée, ces formations semblent correspondre au 3e critère de détaylorisation. Mais compte tenu de l'instabilité du groupe au moment de leur lancement, le critère « durée » est probablement le plus incertain. Réaliser annuellement ou biannuellement des cercles de progrès pour les cadres de proximité d'«objectif cadre ", assurer un suivi rigoureux au poste de travail des gestionnaires d'assurance, dépend, non seulement de la volonté et de la rigueur des personnes directement 
concernées, mais également de la volonté politique des directions de donner à ces personnes le temps nécessaire à l'adaptation et au suivi des formations.

\section{La formation « projet négocié »}

Cette quatrième condition, nécessaire à la détaylorisation concerne tous les partenaires sociaux dès la phase d'initialisation des projets.

Elle n'a pas été effective pour les actions décrites. Mais on peut cependant souligner que les modalités participatives de conception, de réalisation et de suivi des formations, incluant les destinataires des formations, constituent une première étape de négociation où sont levées les barrières entre conception et exécution.

\section{Conclusion}

104 L'expérience relatée et analysée montre, peut être, que la communication interne, qui se cherche encore, dispose à travers les liens qu'elle peut nouer avec la formation et ses pratiques d'une voie possible de développement.

II serait en tout cas intéressant de rechercher dans d'autres entreprises les liens informels et formels qui peuvent exister entre ces deux pratiques et d'observer si se dessine, actuellement, l'amorce d'une évolution leur permettant de s'enrichir mutuellement et de se consolider.

Mais l'enrichissement ne sera réel que si l'évolution va dans le sens d'une détaylorisation et contribue donc à la diffusion de démarches participatives. Sinon, la communication interne, même si elle est épaulée par la formation, retombera dans ses limites actuelles, trop souvent constatées : volontarisme du sommet et unilatéralité ne permettant pas de contribuer au développement de l'autonomie des salariés.

\section{BIBLIOGRAPHIE}

BERNOUX (Ph) : La sociologie des organisations. Seuil 1985. 382 p.

BOUSQUET (N) et GRANDGERARD (C) : « Détaylorisation des formations et stratégies de flexibilité ». Éducation permanente $\mathrm{n}^{\circ}$ 104. (Formation et changement dans les entreprises) - Oct. 1990. pp. 73-82

BOUZON (A) : «L'entreprise aux prises avec sa communication ». Ciném' Action n 63 (Les théories de la communication) - 1992. pp. 185-190.

CHARVIN (F) in SCHWEBIG (Ph) : « La communication interne ». Les communications de l'entreprise. Paris. Ediscience Internationale, ch. 8, pp. 91-103

CROZIER (M) : L'entreprise à l'écoute. InterEditions / Seuil. 1994. p.

Entreprises et Carrières n 251. (Spécial communication interne) - 14-25 juin 1994, p. 
FEUTRIE (M) : Introduction à « Formation et changement dans les entreprises ».Éducation permanente. $\mathrm{n}^{\circ} 104$ - Oct. 1990. pp. 5-7.

FEUTRIE (M) : « Le partage du pouvoir de formation ». Éducation permanente, $\mathrm{n}^{\circ}$ 104.(Formation et changement dans les entreprises) - Oct. 1990. p. 99-108.

IMBERT (M) : « Communication interne, le salarié oublié ». Humanisme et Entreprise - juin 1994.

LE MOENNE (C) : L'ère des communicateurs. Thèse de doctorat d'état en sciences politiques. Université de Rennes 1 - 1990. 424 p.

LE MOENNE (C) : «Communication » by smiling around et «Crise managériale » Réseaux $\mathrm{n}^{\circ} 64$. (Les métiers de la communication) -1994. p. 29-52.

MIEGE (B) : La Société conquise par la communication. PUG -1989. 226 p.

MIEGE (B) : « La nécessaire voie étroite de la recherche ». Ciném'Action n 63. (Les théories de la communication) -1992. pp. 123-129

P. VINAY (O) : « La communication au banc d'essai ». Dynasteurs - Fév. 1990. p. 59-62.

SAINSAULIEU (R) : Sociologie de l'organisation et de l'entreprise. Presses de la Fondation Nationale des Sciences Politiques. Dalloz -1987. 390 p.

SCHWEBIG $(\mathrm{Ph})$ : Les communications de l'entreprise. Paris. Ediscience Internationale $-1988.170 \mathrm{p}$.

\section{NOTES}

1. Nom que nous avons attribué au groupe de manière a en préserver l'anonymat.

2. Le terme acteur est utilisé dans le sens que lui donnent les sociologues des Organisations dans la théorie de l'analyse stratégique BERNOUX $(\mathrm{Ph})$ définit ainsi l'acteur dans son petit vocabulaire de l'analyse stratégique : celui (individu ou groupe) qui participe à l'action et qui a des intérêts communs pour cette action. La Sociologie des organisations, Seuil 1985, p. 166.

3. Selon l'expression de IMBERT (M) : «Communication interne, le salarié oublié », Humanisme et entreprise. Juin 1994

4. IMBERT (M), op.cit.

5. $\operatorname{SCHWEBIG}(\mathrm{Ph})$ : Les communications de l'entreprise Paris - Ediscience Internationale 1988.

p. 15

6. BOUZON (A) : «L'entreprise aux prises avec sa communication ». Ciném'Action $n^{\circ} 63$ (Les théories de la communication). 1992. p. 188.

7. Cette notion sera explicitée dans la 3 partie.

8. Nom attribué à l'organisme de manière à en préserver l'anonymat.

9. CROZIER (M) cité par P. VINAY : «La communication au banc d'essai ». Dynasteurs. Fév. 90. p. 61.

10. SCHWEBIG. (Ph) Les communications de l'entreprise, op. cit. p.15

11. LE MOENNE(C) «Communication «by smiling around » et Crise managériale » Réseaux $n^{\circ} 64$ (Les métiers de la communication) 1994, p. 48.

12. CHARVIN (F), «La communication interne " Les Communications de l'entreprise, op.cit. Ch. 8 , p. 94.

13. Enquête ORC / Contesse citée dans Entreprises et Carrières $n^{\circ} 251$. (Spécial communication interne) - 14-25 juin 1994. p. 6.

14. MIEGE (B) : La société conquise par la communication -PUG 1989. p. 55.

15. SAINSAULIEU (R): Sociologie de l'organisation et de l'entreprise. Presses de la Fondation Nationale des Sciences Politiques. Dalloz-1987. p295 
16. Ibid, p. 300 .

17. Ibid, p. 307.

18. CROZIER (M), L'entreprise à l'écoute Inter Éditions, Seuil. 1994. p. 67.

19. FEUTRIE (M) : Introduction à «Formation et changement dans les entreprises ». Éducation permanente $\mathrm{n}^{\circ} 104$ - Oct. 1990. p. 7.

20. Présentées dans la revue Éducation permanente $n^{\circ} 104$ (Formation et changement dans les entreprises). Oct. 1990.

21. FEUTRIE (M) : « Le partage du pouvoir de formation ». Éducation permanente, op.cit., p. 99.

22. BOUSQUET (N) et GRANDGERARD (C): "Détaylorisation des formations et stratégies de flexibilité ", op.cit., pp. 75-77.

23. BOUSQUET (N) et GRAND-GÉRARD (C), ibid. p. 76.

\section{RÉSUMÉS}

L'expérience relate la mise en place, dans un groupe en pleine restructuration, d'une communication interne participative s'appuyant sur la formation, choisie comme terrain d'expérimentation. L'alliance entre deux acteurs du groupe: les directeurs de ressources humaines et l'organisme de formation permet d'élaborer un nouveau processus de communication aboutissant au renouveau des objectifs et des modalités des actions de formation. La participation, à tous les niveaux, de collaborateurs de l'entreprise, y compris les salariés dits « d'exécution » caractérise ce nouveau processus qu'on peut qualifier de « détaylorisé ».

The experience recounts the setting up. within a group reorganizing its structure of a paticipative in-house communication based on formation a testing ground for training. Union between two elements of this group like the Human Ressources Managers and the training center enables the working out of a new communication process wich leads to the renew of the objectives and the methods of training procedures. The involvement of all the firm's colleagues including the employees, characterizes such a new process which can be qualified as « detaylorised».

\section{AUTEUR}

\section{JACQUELINE CONS-SOUBEIRAN}

L'auteur est diplômée de l'Institut d'Études Politiques de Paris et licenciée en droit. Une partie importante de son activité professionnelle s'est déroulée en entreprise : responsabilités en matière de formation et de communication interne, création d'un cabinet de conseil en communication. Actuellement maître de conférences associée à l'Université de Clermont-Ferrand, ses recherches portent sur l'évolution des pratiques de communication et de formation. 